\title{
Comparison of Methods for Quantification of Topologically Close-Packed Phases in Ni-Based Superalloys
}

\begin{abstract}
A.S. WILSON, K.A. CHRISTOFIDOU, A. EVANS, M.C. HARDY, and H.J. STONE
The ability to quantify accurately the formation of topologically close-packed phases in nickel-based superalloys is key to assessing their thermal stability and ensuring that their performances will not deteriorate during long-term exposure at high temperatures. To investigate the effectiveness of synchrotron XRD for the detection of such minority phases in Ni-based superalloys, the commercial polycrystalline alloy RR1000 was analyzed following exposures of varying times at $800{ }^{\circ} \mathrm{C}$. Data were collected from both solid samples and extracted residues, and additional laboratory X-ray diffraction was performed on the residues. The minor phases were successfully detected in solid samples using synchrotron radiation, and a comparison of the results from these quantification methods shows that the extraction method gives results of the right order of magnitude to reflect the phase quantities that are present in the alloy. However, the results indicate that the synchrotron route is not a suitable method for the quantification of phases present in quantities less than approximately $0.3 \mathrm{wt}$ pct.
\end{abstract}

https://doi.org/10.1007/s11661-019-05442-3

(C) The Author(s) 2019

\section{INTRODUCTION}

POLYCRYSTALLINE nickel-based superalloys are used for hot section components in turbine engines, and therefore experience long periods of time at elevated temperatures during service. These conditions promote the formation of brittle, intermetallic topologically close-packed (TCP) phases that can be detrimental to alloy performance. ${ }^{[1]}$ Mechanisms by which TCP phases have been reported to compromise mechanical performance include interfacial decohesion at precipitate interfaces, ${ }^{[2]}$ brittle fracture of TCP precipitates accelerating crack propagation, ${ }^{[3]}$ and stress concentration at precipitates causing microcrack generation. ${ }^{[4]}$ Historically, TCP phase formation has been a problem for single crystal nickel-based superalloys due to their high refractory contents and $\gamma^{\prime}$ volume fractions. However, new generations of engines present more demanding operational environments for polycrystalline alloys, requiring the development of novel alloys that contain higher refractory element contents and $\gamma^{\prime}$ volume

A. S. WILSON, K. A. CHRISTOFIDOU and H. J. STONE are with the Department of Materials Science and Metallurgy, University of Cambridge, Cambridge CB3 0FS, UK. Contact e-mail: asw33@cam.ac.uk A. EVANS is with the Bundesanstalt für Materialforschung und -prüfung (BAM), 8.5 - Micro-NDT, Unter den Eichen 87, 12205 Berlin, Germany. M. C. HARDY is with the Rolls-Royce plc, PO Box 31, Derby DE24 8BJ, UK.

Manuscript submitted May 24, 2019.

Article published online October 15, 2019 fractions. As such, these alloys are becoming increasingly prone to detrimental TCP phase formation. This has motivated several recent studies to investigate TCP phase formation in polycrystalline alloys. ${ }^{[3,5,6]}$ Such precipitation must generally be avoided, which necessitates balancing the requirement of improved mechanical performance and environmental resistance with the need to avoid compromising the microstructural stability. ${ }^{[7]}$

Key to the assessment of thermal stability during alloy development is the ability to accurately quantify the amount of TCP precipitation that occurs, allowing direct comparison of performance between alloys. A number of different possible methods for performing this quantification can be found in the literature, the first of which is areal analysis. This involves calculating the average area fraction of a particular phase across a large number of images that are representative of the alloy's microstructure, and has previously been used to quantify the precipitation of TCP phases. ${ }^{[8]}$ However, there are various problems with this technique, the most significant of which is the requirement to be able to consistently distinguish the phase of interest from other phases present in the sample. This is not trivial for TCP phases as one type of TCP phase can be similar in morphology and composition to other TCP phases, as well as certain carbides and borides, which makes it difficult to distinguish them using scanning electron microscopy (SEM). Selected area diffraction in the transmission electron microscope (TEM) allows for definitive identification of these phases, but the limited 
size of examinable area precludes the use of this method for the determination of bulk volume fraction.

An alternative method for quantifying constituent phases in a material is to obtain an X-ray diffraction (XRD) pattern and then perform Rietveld refinement. However, due to the exceedingly low volume fractions of TCP phases generally present in commercial Ni-based superalloys, combined with the low symmetry of their crystal structures, it is not possible to reliably detect and characterize these phases using laboratory XRD of a solid sample. As a result, the commonly used quantification method for TCP phases is the electrolytic extraction of the minor phases via preferential dissolution of the $\gamma$ and $\gamma^{\prime}$ phases ${ }^{[9,10]}$ to produce a residue containing a much higher volume fraction of the phases of interest, on which laboratory XRD can be carried out. Combining the results of Rietveld refinement of the obtained diffraction pattern with the mass of the sample before and after extraction, and of the extracted residue, allows the weight fraction of each minor phase in the original material to be calculated.

The extraction method has been used in various studies of TCP formation in Ni-based superalloys. ${ }^{[11-14]}$ However, a number of potential issues have been identified that cast doubt on the reliability of quantitative information obtained using this route. The most significant of these is the potential for loss of TCP precipitates, and other phases, at multiple stages during the extraction and filtration processes. Examples of routes for such loss include the inability to capture particles smaller than the pore size of the filter paper during the filtration process, the potential for unrepresentative sampling of the phases in the residue during transfer to a Si single crystal wafer before XRD, and difficulties in ensuring the complete removal of residue from all of the equipment used for extraction and filtration. In addition, multiple weighing steps must be carried out which all have associated errors. Attempts have been made in previous studies to give an approximate value for the size of the random error associated with such results by repetition of extractions on multiple samples of the same material, with reported values of \pm $0.3 \mathrm{wt} \mathrm{pct}^{[15]}$ and $\pm 0.2 \mathrm{wt} \mathrm{pct}^{[12]}$ These are significant errors given that reported values for TCP phase fractions using this method are generally lower than 2 wt pct, and that phase fractions that are lower than the error values have been reported in both of these studies. The use of a consistent experimental methodology following the ASTM experimental standard E963-95 ${ }^{[9]}$ can minimize some of the aforementioned random errors. However, the capacity for operator and procedural variation, even while adhering to this standard, makes reliable quantitative comparison between results obtained in different studies difficult. In addition, the inability to obtain phase fraction data directly from the solid sample means that it is not known how closely results obtained through the extraction method represent the phase quantities present in the solid material. Thus far in the literature, some of these potential problems with the electrolytic extraction method have been identified, but to date, there has not been any attempt at quantitative evaluation of the accuracy of the results produced using this method.

The high energy and brilliance of synchrotron X-ray radiation allow for the sampling of much larger volumes of material, and the detection of lower volume fractions of phases, than is possible with laboratory XRD. In this study, diffraction patterns from thermally exposed solid samples of the commercial polycrystalline Ni-based superalloy, RR1000, were collected using synchrotron radiation. The aim was twofold: to assess the minimum phase fraction that can be detected and quantified in a solid sample using this method, and to compare these results with the quantities obtained using the standard extraction method.

\section{EXPERIMENTAL PROCEDURE}

The composition of the RR1000 used in this study is given in Table I. The alloy was produced via powder processing by ATI powder metals and supplied by Rolls-Royce plc. The processing route consisted of the production of 270 mesh powder followed by Hot Isostatic Pressing at $1107{ }^{\circ} \mathrm{C}$ and $100 \mathrm{MPa}$ for 4 hours to form a billet. Cylindrical samples of $\sim 5 \mathrm{~mm}$ diameter were obtained from the billet for this work. The $\gamma^{\prime}$ solvus temperature of RR1000 has previously been reported to be $1147^{\circ} \mathrm{C}^{[16]}$ and, therefore, a super-solvus solution heat treatment of 1 hour at $1170{ }^{\circ} \mathrm{C}$ was performed in air. The samples were cooled at a cooling rate of 0.5 to $2{ }^{\circ} \mathrm{C}$ per second from the solution temperature to $900{ }^{\circ} \mathrm{C}$; below $900{ }^{\circ} \mathrm{C}$, the samples were air-cooled.

Long-term isothermal exposures were performed on the RR1000 samples at $800{ }^{\circ} \mathrm{C}$ for durations varying from 15 to 5000 hours, with the material encapsulated in glass ampoules under an Ar atmosphere to minimize oxidation. SEM samples were produced from the material using standard metallographic preparation techniques of mounting in conductive resin, grinding and polishing, culminating with a 20 to 30 minute polish with colloidal silica. The microstructures of these samples were characterized with back scattered electron (BSE) imaging in an FEI Nova NanoSEM and compositional data from the phases present were obtained using energy dispersive X-ray spectroscopy (EDX) with a Bruker detector.

Two types of samples were prepared from the aged material for XRD analysis. Solid cuboidal samples with a cross-sectional width of $\sim 1 \mathrm{~mm}$ were produced by electrodischarge machining (EDM) and the surface layer was then manually ground off to remove any re-cast layer caused by the machining process. The second type of sample was powder consisting of a concentrated residue of minor phases produced from the aged material by electrolytic extraction. Electrolytic phase extractions were performed using an electrolyte of 1 pct tartaric acid in 10 pet $\mathrm{HCl}-m e t h a n o l$ and a current density of $\sim 1 \mathrm{~mA} \mathrm{~mm}{ }^{-2}$ for 6 to 8 hours, following ASTM standard Practice E963-95. ${ }^{[9]}$ The electrolyte was filtered through a polytetrafluoroethylene-type filter with a pore size of $0.45 \mu \mathrm{m}$ using a water aspirator pump to obtain the required residue. The residue was 
Table I. Composition of RR1000 in Atomic Percent

\begin{tabular}{ccccccccccc}
\hline & $\mathrm{Ni}$ & $\mathrm{Cr}$ & $\mathrm{Co}$ & $\mathrm{Mo}$ & $\mathrm{Al}$ & $\mathrm{Ti}$ & $\mathrm{Ta}$ & $\mathrm{C}$ & $\mathrm{B}$ & $\mathrm{Hf}$ \\
\hline RR1000 & 50.5 & 16.5 & 18.4 & 3 & 6.4 & 4.3 & 0.6 & 0.13 & 0.08 & 0.16 \\
\hline \multicolumn{2}{l}{ All elements measured by } & XRF except for B by ICP-OES and C using the combustion infrared detection method. \\
\hline
\end{tabular}

then cleaned using methanol and finally dried in air, and kept covered to prevent contamination.

Synchrotron XRD was performed on the ID22 high-resolution powder diffraction beamline at the European Synchrotron Radiation Facility (ESRF). During data collection, the solid samples were contained in Kapton capillaries, and the extracted powder samples were contained in $1 \mathrm{~mm}$ diameter borosilicate glass capillaries. The capillaries were spun at $787 \mathrm{rpm}$ to reduce any preferred orientation effects on the diffraction data acquired. A multianalyzer stage containing 9 $\mathrm{Si}$ analyzer crystals was used with a beam energy of 50 $\mathrm{keV}$ and a beam size of $1 \mathrm{~mm}$ by $0.9 \mathrm{~mm}$. Data were collected over a $2 \theta$ range of 1 to $34 \mathrm{deg}$ at a scan speed of $0.5 \mathrm{deg} / \mathrm{min}$ for the powder samples and $0.75 \mathrm{deg} / \mathrm{min}$ for the solid samples. Multiple scans were performed for each sample and combined to produce the pattern for analysis.

Laboratory XRD was additionally carried out on the extracted residues using a Bruker D8 DAVINCI diffractometer with a $\mathrm{Cu} \mathrm{K} \alpha$ source and a position-sensitive detector. The extracted residue was deposited on a single-crystal $\mathrm{Si}$ wafer, and the scans were carried out over a range of 10 to $120 \mathrm{deg} 2 \theta$ with a step size of $0.02 \mathrm{deg}$ and dwell time of 2 seconds. Rietveld analysis of the acquired diffraction data was performed using Topas-Academic software. ${ }^{[17]}$ The equilibrium compositions of the phases were predicted by thermodynamic modeling using ThermoCalc ${ }^{[18]}$ software with the database TTNi8. ${ }^{[19]}$

\section{RESULTS}

\section{A. Microstructural Analysis}

SEM BSE images of the microstructure of RR 1000 after thermal exposures of varying durations at $800{ }^{\circ} \mathrm{C}$ are shown in Figure 1. The phases present in these microstructures were identified using their appearance and phase contrast in BSE imaging combined with compositional information from EDX, and their presence was confirmed by analysis of the XRD data. These phases are identified by arrows in the inset in Figure 1(d). It can be seen that after 15 hours at $800^{\circ} \mathrm{C}$ the predominant minority phase is MC-type carbides, which appear bright white in the BSE images and are located primarily at prior particle boundaries. After 1000 hours of exposure at $800{ }^{\circ} \mathrm{C}$, small amounts of both dark $\mathrm{M}_{23} \mathrm{C}_{6}$ phase and bright $\sigma$ phase are present on the grain boundaries, alongside a small quantity of MC carbides. Following both 2525 and 5000 hours of exposure at $800{ }^{\circ} \mathrm{C}$, a higher fraction of larger discrete $\sigma$ precipitates can be seen on the grain boundaries, alongside the other phases. The compositional difference between the $\sigma$ and $\mathrm{M}_{23} \mathrm{C}_{6}$ precipitates is illustrated in Figure 2 and quantified in Table II for the alloy following exposure for 5000 hours at $800{ }^{\circ} \mathrm{C}$. It can be seen that the $\sigma$ precipitate is primarily enriched in Mo as well as Cr compared with the surrounding matrix phase, whereas the $\mathrm{M}_{23} \mathrm{C}_{6}$ precipitate is enriched primarily in $\mathrm{Cr}$ with a lower level of Mo. The given $\mathrm{M}_{23} \mathrm{C}_{6}$ composition does not include $\mathrm{C}$ as $\mathrm{C}$ cannot be measured accurately using EDX but if the stoichiometrically expected $\mathrm{C}$ content is included then the compositional trends observed with respect to the $\sigma$ phase remain the same. An additional difference between the two phases is that the $\mathrm{M}_{23} \mathrm{C}_{6}$ phase is depleted in $\mathrm{Co}$, whereas the $\sigma$ phase contains $\mathrm{Co}$ at similar levels to those found in the matrix.

\section{B. XRD Data Analysis}

Initial Rietveld refinements were performed on the synchrotron XRD data for the extracted material. The structural and site occupancy information obtained was subsequently used as the starting point for the refinement of the synchrotron data from the solid samples and the laboratory XRD data from the extracted material. The phases included in the refinements were the $\gamma$ and $\gamma^{\prime}$ phases as well as the minor phases, $\mathrm{M}_{23} \mathrm{C}_{6}, \sigma$ and TiC, as identified using SEM. In addition, $\mathrm{HfO}_{2}$ was identified in the XRD spectra, consistent with previous reports of $\mathrm{HfO}_{2}$ formation in RR1000. ${ }^{[12]}$

The compositions used in the Rietveld refinement for the $\gamma$ and $\gamma^{\prime}$ phases were those predicted for this alloy at $800{ }^{\circ} \mathrm{C}$ using ThermoCalc software with the database TTNi8 (see Table III). For the $\gamma^{\prime}$ phase, this was combined with information from Stoloff ${ }^{[20]}$ concerning which elements occupy which type of site within the ordered structure. $\mathrm{Ni}$ and Co were allocated solely to the $\mathrm{Ni}$ site; Ti, Ta, and Al only to the Al site; and $\mathrm{Cr}$ and Mo, which can occupy both sites, were distributed between them to ensure all site occupancies were equal to 1 . In both the $\gamma$ and $\gamma^{\prime}$ phases, the site occupancies were not allowed to refine, as this made the refinement unstable.

In addition, the compositions of the $\mathrm{M}_{23} \mathrm{C}_{6}$ and $\sigma$ phases as obtained by EDX on samples following exposure for 5000 hours at $800{ }^{\circ} \mathrm{C}$ were used in the refinements (see Table II). In both phases, the levels of $\mathrm{Al}$, Ti and Ta detected were very low (below 3.5 at. pct) and were considered likely to be the result of X-rays originating in the surrounding $\gamma^{\prime}$ phase. Therefore, it was decided not to include these in the analysis, and the relative amounts of the remaining elements $(\mathrm{Ni}, \mathrm{Co}, \mathrm{Cr}$, and Mo) were re-scaled. This decision is supported by compositional data collected using atom probe tomography found in the literature, which show very limited 

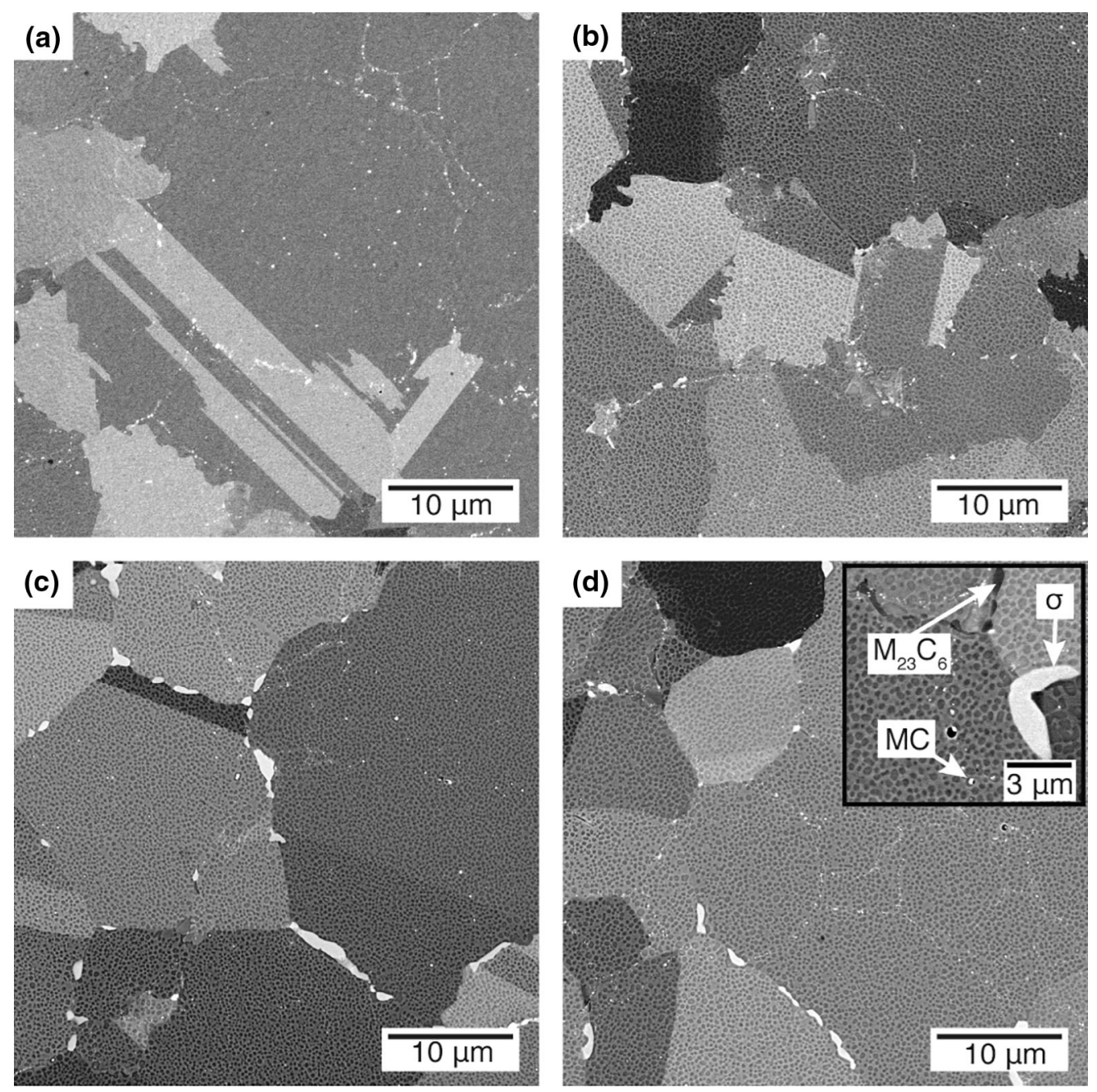

Fig. 1-BSE SEM images of the microstructures of RR1000 after exposure at $800{ }^{\circ} \mathrm{C}$ for $(a) 15 \mathrm{~h},(b) 1000 \mathrm{~h},(c) 2525 \mathrm{~h}$, and $(d) 5000 \mathrm{~h}$. The $\mathrm{MC}, \mathrm{M}_{23} \mathrm{C}_{6}$, and $\sigma$ phases are identified in the inset in $(d)$.
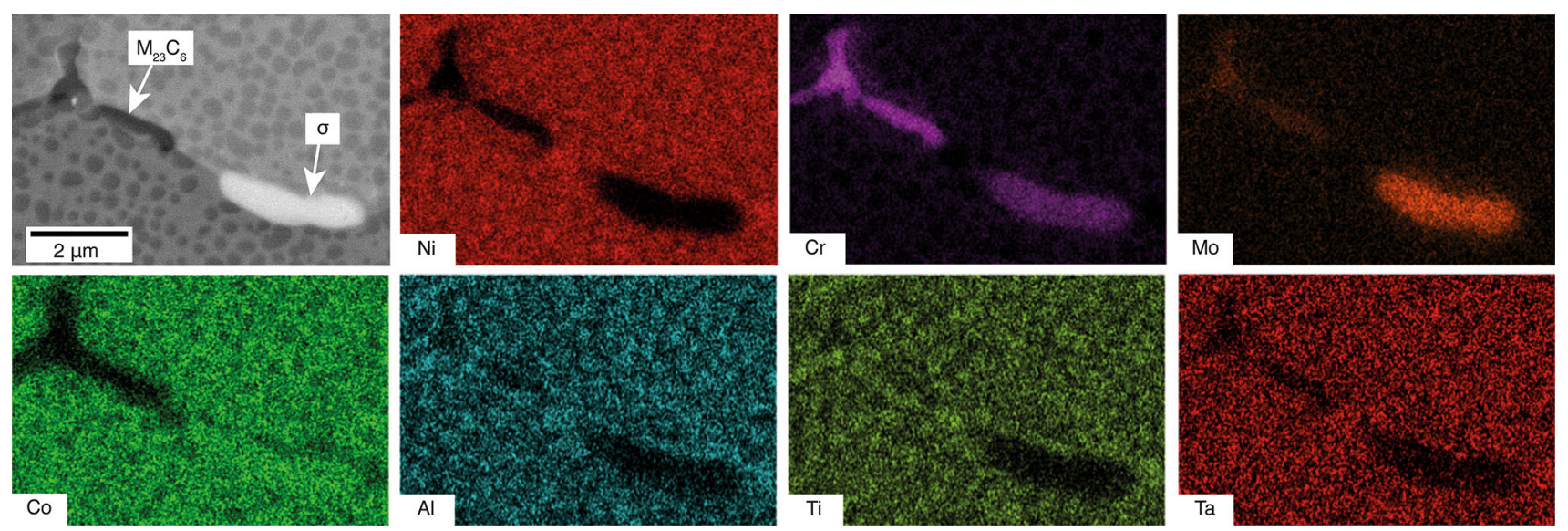

Fig. 2-BSE SEM image of a grain boundary in RR1000 following exposure for $5000 \mathrm{~h}$ at $800{ }^{\circ} \mathrm{C}$ along with the associated SEM-EDX elemental distribution maps. The $\mathrm{M}_{23} \mathrm{C}_{6}$ and $\sigma$ phases are identified in the BSE SEM image.

solubility for $\mathrm{Al}$ and $\mathrm{Ta}$ in the $\sigma$ phase $^{[5]}$ and no solubility for $\mathrm{Al}$ and $\mathrm{Ti}$ in the $\mathrm{M}_{23} \mathrm{C}_{6}$ phase. ${ }^{[21]}$ The $\mathrm{M}_{23} \mathrm{C}_{6}$ structure contains 4 non-equivalent metal atom positions (see Table IV), so the atoms were distributed evenly between these in proportion with the number of each type of site in a unit cell and the overall phase 
Table II. $\sigma$ and $\mathrm{M}_{23} \mathrm{C}_{6}$ Compositions in Atomic Percent Obtained by EDX from a Polished Specimen Following Thermal Exposure for $5000 \mathrm{~h}$ at $800{ }^{\circ} \mathrm{C}$ (The Reported Error is the Standard Deviation of 10 Measurements Made Across a Selection of Precipitates of the Relevant Phase)

\begin{tabular}{lccrrrrr}
\hline & $\mathrm{Ni}$ & $\mathrm{Cr}$ & \multicolumn{1}{c}{$\mathrm{Co}$} & \multicolumn{1}{c}{ Mo } & \multicolumn{1}{c}{$\mathrm{Al}$} & $\mathrm{Ti}$ & $\mathrm{Ta}$ \\
\hline $\mathrm{M}_{23} \mathrm{C}_{6}$ & Bal. & $54.2 \pm 6.0$ & $8.7 \pm 1.2$ & $6.1 \pm 0.5$ & $2.3 \pm 0.6$ & $3.4 \pm 0.6$ & $0.3 \pm 0.1$ \\
$\sigma$ & Bal. & $36.1 \pm 0.4$ & $22.7 \pm 0.2$ & $10.7 \pm 0.1$ & $1.6 \pm 0.0$ & $2.4 \pm 0.0$ & $0.1 \pm 0.0$ \\
\hline
\end{tabular}

Table III. $\gamma$ and $\gamma^{\prime}$ Phase Compositions at $800{ }^{\circ} \mathrm{C}$ Predicted Using ThermoCalc with Database TTNi8 (Atomic Percent)

\begin{tabular}{lccccccc}
\hline & $\mathrm{Ni}$ & $\mathrm{Cr}$ & $\mathrm{Co}$ & $\mathrm{Mo}$ & $\mathrm{Al}$ & $\mathrm{Ti}$ & $\mathrm{Ta}$ \\
\hline$\gamma$ & 42.6 & 26.2 & 25.2 & 3.6 & 2.0 & 0.3 & 0 \\
$\gamma^{\prime}$ & 65.7 & 1.8 & 9 & 0.3 & 12.2 & 9.6 & 1.4 \\
\hline
\end{tabular}

Table IV. Crystal Structural Information for $\mathrm{M}_{23} \mathrm{C}_{6}$ Taken from Medvedeva et al. ${ }^{[22]}$ with Atomic Positions Taken from CIF File ICSD-154719

\begin{tabular}{lcllllc}
\hline Site & $\begin{array}{c}\text { Wyckoff } \\
\text { Position }\end{array}$ & \multicolumn{1}{c}{$x$} & \multicolumn{1}{c}{$y$} & \multicolumn{1}{c}{$z$} & $\begin{array}{c}\text { Coordination } \\
\text { Number }\end{array}$ \\
\hline M1 & $4 \mathrm{a}$ & 0 & 0 & 0 & 12 \\
M2 & $8 \mathrm{c}$ & 0.25 & 0.25 & 0.25 & 16 \\
M3 & $32 \mathrm{f}$ & 0.38 & 0.38 & 0.38 & 13 \\
M4 & $48 \mathrm{~h}$ & 0 & 0.171 & 0.171 & 14 \\
C & $24 \mathrm{e}$ & 0.272 & 0 & 0 & 8 \\
\hline
\end{tabular}

Table V. Crystal Structural Information for $\sigma$ Taken from Joubert $^{[25]}$ with Atomic Positions Taken from Yakel ${ }^{[24]}$

\begin{tabular}{lcllllc}
\hline Site & $\begin{array}{c}\text { Wyckoff } \\
\text { Position }\end{array}$ & \multicolumn{1}{c}{$x$} & \multicolumn{1}{c}{$y$} & \multicolumn{1}{c}{$z$} & $\begin{array}{c}\text { Coordination } \\
\text { Number }\end{array}$ \\
\hline A & $2 \mathrm{a}$ & 0 & 0 & 0 & 12 \\
B & $4 \mathrm{f}$ & 0.39800 & 0.39800 & 0 & 15 \\
C & $8 \mathrm{i} 1$ & 0.46371 & 0.13057 & 0 & 14 \\
D & $8 \mathrm{i} 2$ & 0.73868 & 0.06572 & 0 & 12 \\
E & $8 \mathrm{j}$ & 0.18302 & 0.18302 & 0.2512 & 14 \\
\hline
\end{tabular}

composition. These site occupancies were allowed to refine while the total site occupancy of each site was constrained to be equal to 1 and the overall composition was constrained to be equal to the measured composition.

The crystal structure of the $\sigma$ phase has the $\mathrm{P} 42 / \mathrm{mnm}$ space group and contains 5 non-equivalent positions with coordination numbers varying from 12 to 15 (see Table V). It is known that Group Vb or VIb atoms that are deficient in electrons and usually have larger radii preferentially occupy the sites with a coordination number of 15 , while the electron-rich, smaller atoms from Group VIIb or VIII prefer to occupy sites with a coordination number of $12 .{ }^{[23]}$ As a result of these ordering preferences, the initial site occupancies were calculated by allocating all of the Mo and $\mathrm{Cr}$ to the 14 and 15 coordinated sites, whereas the $\mathrm{Ni}$ and $\mathrm{Co}$ were allocated primarily to the 12 coordinated sites and then to fill the other sites as required. The site occupancies were then allowed to refine for all elements on each site, under the constraints of each site occupancy being equal to 1 and the total composition remaining equal to the measured composition. The atomic positions used to start the refinement were taken from Yakel ${ }^{[24]}$ for an Fe-, Cr-, Ni-, Mo-, and Mn-containing $\sigma$ phase (see Table V). Seven atomic position parameters were allowed to refine as detailed by Yakel. ${ }^{[24]}$ The compositions and atomic positions for $\mathrm{TiC}$ and $\mathrm{HfO}_{2}$ were kept fixed.

Isotropic temperature factors were refined for each phase as refining individual temperature factors for each atom caused very large errors in the values returned. A stable refinement was obtained while allowing lattice parameters, peak shapes, and temperature factors to refine for all phases and also refining site occupancies for $\mathrm{M}_{23} \mathrm{C}_{6}$ and $\sigma$ and atomic positions for the $\sigma$ phase for the synchrotron data acquired from the 1000-hour, 2525-hour, and 5000-hour-extracted residues.

The synchrotron data obtained from the 5000 hours at $800{ }^{\circ} \mathrm{C}$ extracted residue are displayed in Figure 3(a), along with the calculated fit and the residual. The residual is the difference between the experimental data and the calculated fit, and therefore its relatively small size shows that a good fit to the experimental data has been obtained. For the primary region of interest, shown in Figure 3(b), the peaks are labeled according to which phase they originate from. All major peaks have been identified and fitted, although it was not possible to identify the origin of several minor peaks. The $\mathrm{M}_{23} \mathrm{C}_{6}, \sigma$, and $\gamma / \gamma^{\prime}$ peaks are clearly identifiable and can be fitted with a large degree of confidence, but the MC peaks are both broad and difficult to separate from peaks of other phases, causing larger errors to be obtained in the fits of this phase. However, the predominant minor phase in the sample exposed for 15 hours is MC carbide (see Figure 1(a)) and, therefore, in data from the extract of this sample, the MC peaks are more intense and well defined. As a result, MC phase information obtained from fitting the data for the 15 -hour sample was used to inform the fits for the data from the longer duration samples. It should be noted that, while only one peak has been labeled as resulting from the MC phase in the displayed region of the spectrum in Figure 3(b), overall more than ten peaks were fitted from this phase. Table VI gives the lattice parameters and atomic coordinates for the $\sigma$ phase and the $\mathrm{M}_{23} \mathrm{C}_{6}$ phase, which were obtained from Rietveld refinement of the synchrotron X-ray data from the extracted residue of the 5000-hour 


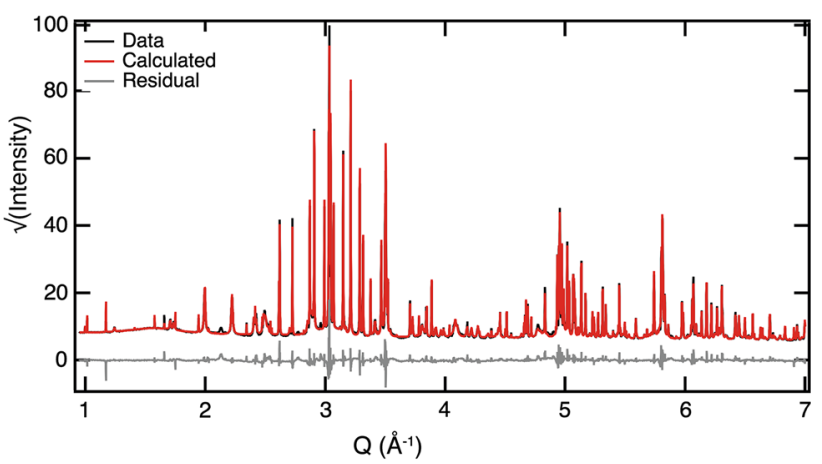

(a)

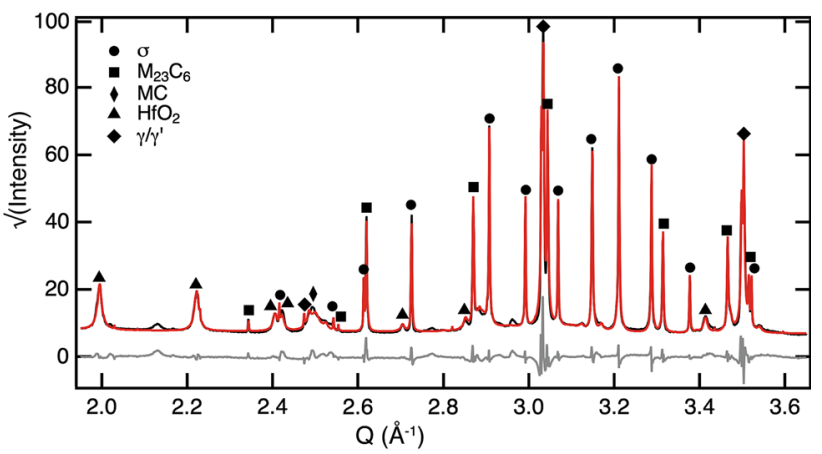

(b)

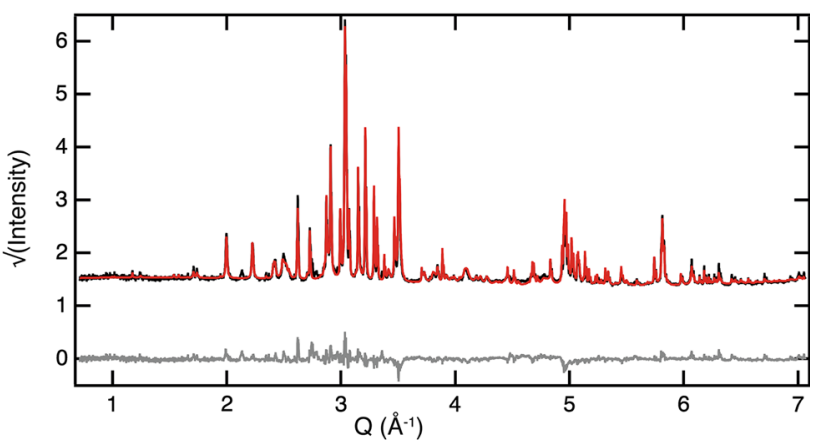

(c)

Fig. 3-X-ray diffraction (XRD) data for the extracted residue after thermal exposure for $5000 \mathrm{~h}$ at $800{ }^{\circ} \mathrm{C}$, displayed with the calculated fit and the residual. (a) Data collected with synchrotron XRD, $(b)$ a magnified view of a selected angular range of the synchrotron XRD data with phase identification markers, and $(c)$ data collected with laboratory XRD.

sample. The laboratory X-ray data obtained from the extracted residue of the same specimen is shown in Figure 3(c). This data is comparable to the synchrotron X-ray data but is not of quite such a high quality. It should be noted that in order to obtain a good fit to the laboratory X-ray data it was necessary to use the parameters obtained from refining the higher quality synchrotron data as the starting point for the refinement.

Fitting the data obtained from the solid samples was more challenging for several reasons. The background contained a large number of humps of various sizes, which had to be modeled using individual pseudo-voigt peaks. It is suggested that the source of these humps could be stray scatter from the sample passing between the crystals in the analyzer and reaching the detector. This is more of a problem at high beam energies, such as used in this experiment, as the analyzer crystals are at a low angle with respect to the diffracted beams. Also, it is possible that the presence of short-range order in these phases may contribute to these background features. In addition, the peaks from the minor phases of interest were of sufficiently low intensities that individual peaks had to be fitted separately and then the parameters for that phase fixed in order to obtain a stable refinement.

The synchrotron X-ray data obtained from the 5000 hours at $800{ }^{\circ} \mathrm{C}$ solid sample is shown in Figure 4. As expected from the relative fractions of the phases in the sample, the intensity of the peaks corresponding to the minor phases is very weak compared to the intensity of the $\gamma / \gamma^{\prime}$ peaks. Closer examination of the region of the spectrum displayed in Figure 4(b) reveals that the majority of minor peaks were correctly fitted but it must be noted that the fit obtained to both the peaks and the background is inferior to that obtained for the data from the extracted residue. Figure 5 combines the spectra obtained from the solid specimens into one plot where they are stacked and slightly offset for ease of visualization. It can clearly be seen that the peaks associated with the MC phase are present in the 15-hour specimen, but then gradually reduce in intensity after longer durations, whereas the $\mathrm{M}_{23} \mathrm{C}_{6}$ and $\sigma$ peaks are not visible after 15 hours but appear in the 1000-hour specimen and then increase in intensity for the 2525-hour and 5000-hour specimens. In the spectrum for the 1000-hour specimen, some of these minor phase peaks can be distinguished by eye, but the majority cannot be fitted, indicating that the phase fractions are too low to be reliably quantified in the 1000 -hour solid sample using this technique.

Quantitative results for the weight fractions of a selection of the minor phases obtained by Rietveld refinement from the diffraction data are displayed in Figure 6 and detailed in Table VII. According to the results obtained from the solid samples, the quantities of the $\sigma$ and $\mathrm{M}_{23} \mathrm{C}_{6}$ phases increase with the increasing exposure time, and the quantity of MC carbides decreases (as they are only detectible in the 15-hour specimen). Comparing the results of the two measurement methods on the extracted residues shows very similar results for the $\sigma$ and $\mathrm{M}_{23} \mathrm{C}_{6}$ phases although they diverge somewhat for the MC phase, particularly for low exposure durations. Comparing the results from the solid samples and the extracted residues reveals mostly the same trends for all of the phases. While the trends are reproduced, some differences in the magnitude of the values obtained via the different methods can be seen. In addition, an unexpected decrease in $\sigma$ precipitation between 2525 hours and 5000 hours of exposure is observed in the results from the extracted residue. To investigate this discrepancy, image analysis of BSE images of the microstructures of the 2525 hour- and 5000-hour-exposed specimens was performed to determine the area fraction of $\sigma$. An area of $0.92 \pm 0.06 \mathrm{pct}$ was found for the 2525-hour specimen and $0.69 \pm$ 0.04 pct for the 5000-hour specimen, where the reported 
Table VI. Lattice Parameters and Atomic Coordinates for the $\sigma$ and $\mathrm{M}_{23} \mathrm{C}_{6}$ Phases Obtained from Refinement of the Synchrotron X-ray Diffraction Data for the Extracted Residue of the Sample Thermally Exposed for $5000 \mathrm{~h}$ at $800{ }^{\circ} \mathrm{C}$

\begin{tabular}{lllll}
\hline & & \multicolumn{3}{l}{ Atomic Coordinates } \\
\cline { 3 - 5 } Phase & Lattice Parameters $(\AA)$ & $x$ & \multicolumn{1}{c}{$y$} & \multicolumn{1}{c}{$z$} \\
\hline$\sigma$ & $a=8.91$ & 0 & 0 & 0 \\
& $c=4.61$ & 0.396 & 0.396 & 0 \\
& & 0.464 & 0.128 & 0 \\
& & 0.74 & 0.066 & 0 \\
$\mathrm{M}_{23} \mathrm{C}_{6}$ & $a=10.72$ & 0.183 & 0.183 & 0.248 \\
& & 0 & 0 & 0 \\
& & 0.25 & 0.25 & 0.25 \\
& & 0.38 & 0.38 & 0.38 \\
& & 0 & 0.171 & 0.171 \\
& & 0.272 & 0 & 0 \\
\hline
\end{tabular}

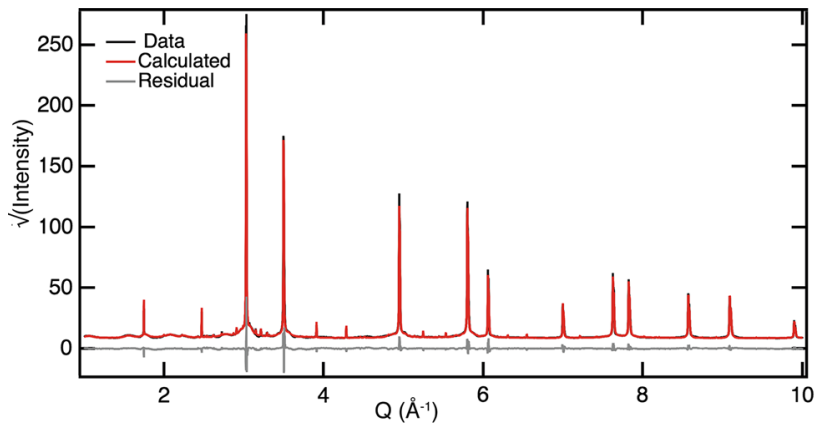

(a)

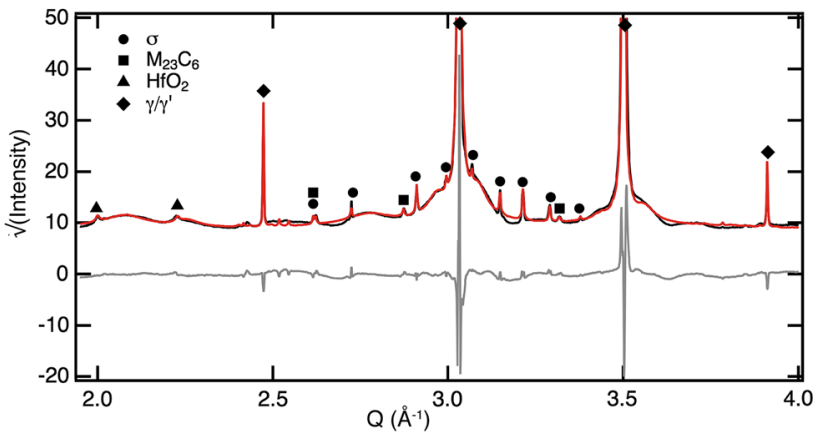

(b)

Fig. 4-Synchrotron X-ray diffraction data for the solid sample after thermal exposure for $5000 \mathrm{~h}$ at $800{ }^{\circ} \mathrm{C}$, displayed with the calculated fit and the residual. (a) The complete angular range over which refinement was performed and $(b)$ a magnified view of a selected angular range with phase identification markers.

error is the standard error obtained from image analysis of 30 randomly selected regions for each sample. These results appear to support the unexpected trend seen in the results obtained from the extracted residues. However, consideration of the relative intensity of the $\sigma$ peaks in the solid spectra for the 2525- and 5000-hour samples reveals a clear increase in their intensity

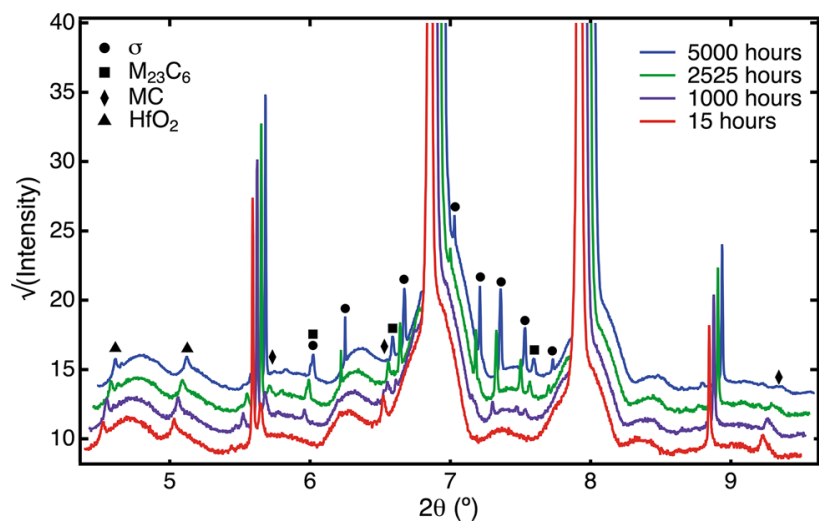

Fig. 5- Synchrotron XRD patterns for solid samples following various thermal exposure durations at $800{ }^{\circ} \mathrm{C}$. The patterns are offset vertically and horizontally for ease of visualization such that the numbers on the axes apply only to the 15 -h spectra.

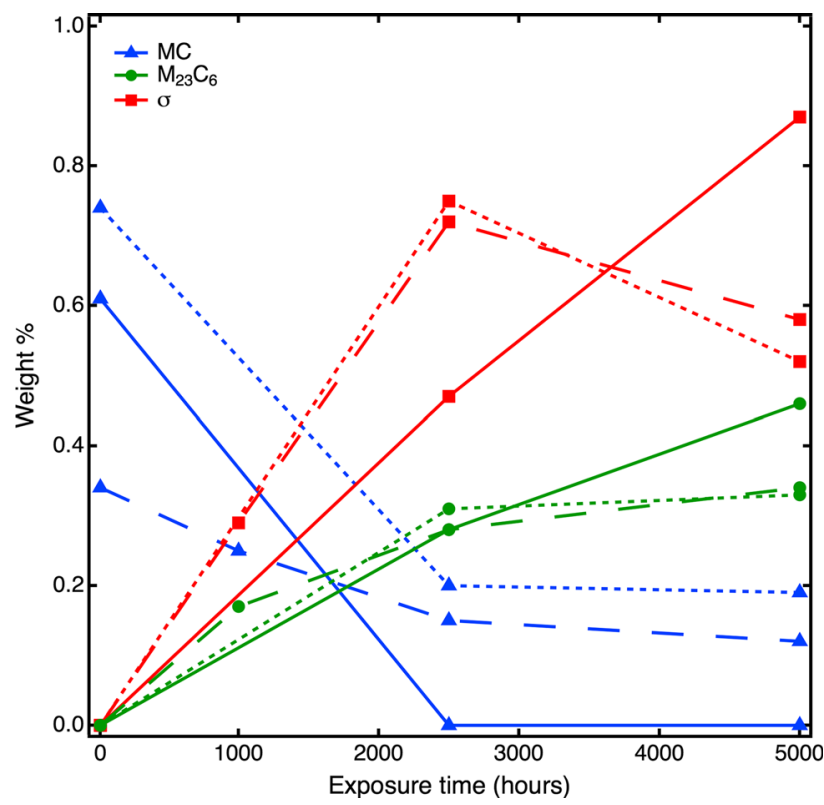

Fig. 6-Weight Percent of minor phases in RR1000 at $800^{\circ} \mathrm{C}$ calculated from data collected by different experimental methods (solid line $=$ solid sample measured using synchrotron XRD, dashed line $=$ extracted sample measured using synchrotron XRD, dotted line $=$ extracted sample measured using laboratory XRD).

between 2525 and 5000 hours, while the intensity of the $\gamma / \gamma^{\prime}$ peaks is comparable across the two spectra.

\section{DISCUSSION}

\section{A. Assessment of Results Obtained by Different Methods}

The majority of the quantitative results obtained from diffraction analysis show an increase in $\sigma$ and $\mathrm{M}_{23} \mathrm{C}_{6}$ precipitation (Figure 6), which agrees with the observations made using SEM analysis (Figure 1). A notable exception to this trend is the decrease in the quantity of the $\sigma$ phase fraction obtained between 2525 and 5000 hours of exposure in the data presented from the extracted residues. This is unexpected and disagrees with the 
Table VII. Quantitative Data for Weight Percent of Phases Obtained from Rietveld Refinement of the Diffraction Data for All Three Acquisition Methods

\begin{tabular}{clccc}
\hline Exposure Duration (h) & XRD Method & $\sigma$ & $\mathrm{M}_{23} \mathrm{C}_{6}$ & $\mathrm{MC}$ \\
\hline \multirow{2}{*}{5000} & synchrotron (s) & 0.87 & 0.46 & 0.00 \\
& synchrotron (e) & 0.58 & 0.34 & 0.12 \\
\multirow{2}{*}{2525} & laboratory (e) & 0.52 & 0.33 & 0.19 \\
& synchrotron (s) & 0.47 & 0.28 & 0.00 \\
& synchrotron (e) & 0.72 & 0.28 & 0.15 \\
1000 & laboratory (e) & 0.75 & 0.31 & 0.20 \\
15 & synchrotron (e) & 0.29 & 0.17 & 0.25 \\
& synchrotron (s) & 0.00 & 0.00 & 0.61 \\
& synchrotron (e) & 0.00 & 0.00 & 0.34 \\
& laboratory (e) & 0.00 & 0.00 & 0.74 \\
\hline
\end{tabular}

Data collected from solid sample (s) or extracted residue (e).

results obtained from the solid samples, which show the continuation of the expected trend of increasing $\sigma$ precipitation with time. A possible explanation of this behavior would be the metastability of the $\sigma$ phase, however, no evidence to support this has been found. Examination of the relative $\sigma$ peak intensities in the spectra from the 2525- and 5000-hour solid samples confirms that there is an increase in the quantity of the $\sigma$ phase in these samples. While the area analysis results suggest a decrease in phase fraction in the SEM samples, the possible inaccuracy of this measurement method when the phase fractions in both samples are so similar makes it a less compelling piece of evidence. Therefore, the apparent decrease in $\sigma$ precipitation shown by analysis of the extracted residues could be an example of the possible problems and inaccuracies with using the extraction method for quantitation of very small quantities of these phases in commercial alloys. Alternatively, it is possible that this behavior is associated with sample-to-sample variations, as errors of $\pm 0.3 \mathrm{wt} \mathrm{pct}^{[15]}$ and $\pm 0.2 \mathrm{wt} \mathrm{pct}^{[12]}$ for phase fractions have been reported for this in the literature. However, it was not possible to investigate this further in the current study due to limitations on the availability of synchrotron beam time.

The values of the phase fractions obtained by these different measurement methods are encouragingly similar in magnitude (see Figure 6). This is particularly apparent for the $\mathrm{M}_{23} \mathrm{C}_{6}$ phase and suggests that the extraction route can provide quantitative results, which are representative of the phase fractions present in the solid material. This additionally implies that there is not a significant amount of precipitate loss during the various stages of the extraction and filtration processes. However, the size of the variation between the results obtained by the different methods for the MC and $\sigma$ phases is significant given the very low phase fractions we are considering in this material. Therefore, the repetition of this experiment using a less stable alloy, which precipitates much larger quantities of $\sigma$ after long exposure times, may be warranted for a more conclusive assessment. The presence of larger quantities of the minor phases would allow more accurate fitting of the data obtained from the solid samples and thus better quantification of the accuracy of the results obtained from the extraction method.

Comparison of the results from synchrotron XRD of the solid samples with those reported from a previous investigation into the stability of RR1000 by Hunt ${ }^{[12]}$ reveals a considerable discrepancy in the phase fractions obtained. Hunt reported significantly higher $\sigma$ phase fractions following the same exposure conditions using the method of electrolytic extraction and laboratory XRD. For example, a $\sigma$ fraction of $\sim 4.6 \times$ larger than that found in this study ( $2.15 \mathrm{wt}$ pct) after $\sim 2500$ hours at $800{ }^{\circ} \mathrm{C}$ was reported. Comparison of the material and experimental conditions reported by Hunt to those used in the current study reveals one difference, which is the prior processing of the material and resultant grain size. The current study was performed on hot isostatically pressed material after 15 hours at $800{ }^{\circ} \mathrm{C}$, whereas Hunt used forged material after 16 hours at $800{ }^{\circ} \mathrm{C}$. Assuming equiaxed grains, this results in a $1.44 \times$ larger theoretical grain boundary density in Hunt's material, calculated from grain size measurements according to ASTM standard method E112-13. ${ }^{[26]}$ Grain boundaries provide preferential sites for $\sigma$ precipitation so a higher grain boundary density could result in more $\sigma$ precipitation explaining part of the observed discrepancy.

\section{B. Site Occupancies in the $\sigma$ and $M_{23} C_{6}$ Phases}

Information about preferential elemental segregation to different sites within the $\sigma$ and $\mathrm{M}_{23} \mathrm{C}_{6}$ phases can be obtained following the refinements. While the exact values obtained vary with repeated refinements of the data, some significant preferential elemental segregation can be observed consistently for both phases. The final site occupancies obtained for the $\mathrm{M}_{23} \mathrm{C}_{6}$ phase differ considerably from the originally assigned random distribution of the elements in proportion to the overall phase composition. A significant difference is the segregation of the majority of the Mo content to site M2, which has the highest coordination number of 16 . This may be explained by the relative atomic sizes of the elements involved; $\mathrm{Ni}, \mathrm{Co}$ and $\mathrm{Cr}$ are all of similar sizes, but Mo is significantly larger, hence its preference for the higher coordinated site. The other incidence of severe segregation of an element to a site was found to be $\mathrm{Cr}$ segregating to site M3 but a mechanistic origin for this has not yet been identified.

For the $\sigma$ phase, the most significant observation is the preferential distribution of Mo to site B. Once again, this can be explained by the relative atomic sizes of the elements as Mo is the largest element and, therefore, preferentially occupies the B site, which has the largest coordination number of 15 . This effect has previously been reported by Yakel. ${ }^{[24]}$

\section{Factors Impacting the Quality of Data Fitting}

A good fit was obtained to the synchrotron data acquired from all of the samples except the 1000-hour exposure, particularly for the extracted residues. This is shown by the small size of the residual produced by 
Rietveld refinement of the data (see Figure 3). However, when assessing this quantification technique it is important to consider any factors that may have impacted the quality of the obtained fits and thus the accuracy of the reported phase fractions. These factors include the phase compositions used and the presence of a small number of unidentified peaks and will be discussed below.

The $\sigma$ and $\mathrm{M}_{23} \mathrm{C}_{6}$ compositions used for analysis of the data for all of the specimens were obtained by SEM-EDX on a polished sample after thermal exposure for 5000 hours at $800{ }^{\circ} \mathrm{C}$ (Table II). Compositions were obtained from the alloy in this condition because it contained the largest precipitates of these phases and thus minimized the complications that may arise as a result of the interaction volume from which X-rays were detected including surrounding phases such as $\gamma$ and $\gamma^{\prime}$. However, the thickness of the precipitates is not known, so there is always the potential for compositional contamination from phases below the precipitate. To determine whether the compositions of these phases vary significantly depending on the exposure time, they were also measured in the material exposed for 2525 hours using the same method. The compositions obtained were very similar for both phases, with the only significant difference being an apparent change in the Ni:Co ratio. Using the compositions obtained from the 2525-hour-exposed sample as alternative constraints on the site occupancies for the 2525-hour-extracted sample data produced, no significant improvement in the fit compared to that obtained using the 5000-hour compositions. The high quality of the fit obtained to the $\sigma$ and $\mathrm{M}_{23} \mathrm{C}_{6}$ peaks in all of the data sets using the compositions obtained by EDX from the 5000-hour sample meant that these data were judged to be sufficiently representative of the true composition for the required purpose.

A small number of low intensity peaks in the data for the 5000-hour extract have not been accounted for by the identified phases (see Figure $3 b$ ) and similar occurrences can be seen in all of the data sets, although the peak positions are not consistent across all of the spectra. It has not been possible to identify the phases which these peaks are originating from so this will have a small effect on the calculated quantities of the identified phases. However, the relatively small size of the peaks involved suggests that this effect is likely to be minimal.

\section{Synchrotron XRD of solid samples as a route for TCP quantitation}

Some useful conclusions can be drawn from these results as to the lower limits on the quantity of minor phases that can be detected using this method, as well as the factors that affect these limits. Considering the values obtained from refinement in Table VII, we can see that the phase fraction required for detection in the solid sample depends on the phase involved. For example, $\sigma$ levels that are expected from the extraction results to be $\sim 0.3 \mathrm{wt}$ pct cannot be quantified in the solid 1000-hour sample but $\mathrm{M}_{23} \mathrm{C}_{6}$ levels of $\sim 0.3 \mathrm{wt}$ pct can in the solid 2525-hour sample. This quantification limit is also affected by whether the peaks are isolated or in a region containing many other phase peaks as well as the broadness of the peaks. These last two factors explain why the MC carbides can only be quantified in the 15-hour solid sample and not in the solid material following longer durations. The MC peaks are very broad compared to those of the other phases and some of them coincide with peaks of the $\sigma$ and $\mathrm{M}_{23} \mathrm{C}_{6}$ phases, which obscure these peaks and makes them difficult to fit accurately. The 15-hour solid sample contains a higher fraction of the $\mathrm{MC}$ phase and does not contain the $\sigma$ and $\mathrm{M}_{23} \mathrm{C}_{6}$ phases, allowing the $\mathrm{MC}$ phase to be fitted with a high degree of confidence. Overall, we can see that, given the right phase and combination of other phases present, phase fractions as low as $\sim 0.3 \mathrm{wt}$ pct can be quantified in solid material for some phases using this method, showing the power of this technique for detecting small quantities of phases.

\section{CONCLUSIONS}

The measurement route used here involving synchrotron diffraction of solid specimens allows for the detection of very low phase fractions $(\sim 0.3 \mathrm{wt}$ pct $)$ of minor phases in a nickel-based superalloy. The exact detection limit depends on the phase under consideration as it is affected by various factors including the positions of the peaks relative to the other peaks in the data and the peak breadths.

The phase fractions obtained for the solid and extracted samples were of a similar order of magnitude providing encouraging evidence that the results produced by the extraction method are generally representative of the phases present in the material. The low phase fractions of $\sigma$ present in all of the RR 1000 samples prevented a more detailed assessment of the accuracy of the method from being made. However, it can be concluded that synchrotron diffraction analysis of solid samples is not a suitable route for the quantification of phases present in quantities below $\sim 0.3 \mathrm{wt}$ pct in commercial alloys, as these result in peaks with extremely low intensities that can be difficult to fit with confidence.

\section{ACKNOWLEDGMENTS}

This work was supported by the Rolls-Royce EPSRC Strategic Partnership under EP/M005607/1, $\mathrm{EP} / \mathrm{H} 022309 / 1$ and $\mathrm{EP} / \mathrm{H} 500375 / 1$. The underlying research data can be found at https://doi.org/10.17863/ CAM. 44528 .

\section{OPEN ACCESS}

This article is distributed under the terms of the Creative Commons Attribution 4.0 International License (http://creativecommons.org/licenses/by/4.0/), 
which permits unrestricted use, distribution, and reproduction in any medium, provided you give appropriate credit to the original author(s) and the source, provide a link to the Creative Commons license, and indicate if changes were made.

\section{REFERENCES}

1. R. Reed: The Superalloys Fundamentals and Applications, Cambridge University Press, Cambridge, 2006, pp. 51-53.

2. Q. Shi, X. Ding, J. Chen, X. Zhang, Y. Zheng, and Q. Feng: Metall. Mater. Trans. A, 2014, vol. 45A, pp. 1665-69.

3. Y. Liu, Y. Wu, M. Kang, M. Wang, M. Li, H. Gao, J. Wang, B. Sun, and Y. Ning: Mater. Sci. Eng. A, 2018, vol. 736A, pp. $438-52$.

4. Z. Zhang and Z. Yue: J. Alloys Compd., 2018, vol. 746, pp. 84-92.

5. S. Antonov, J. Huo, Q. Feng, D. Isheim, D.N. Seidman, R.C. Helmink, E. Sun, and S. Tin: Mater. Sci. Eng. A, 2017, vol. $687 \mathrm{~A}$, pp. $232-40$.

6. R. Krakow, D.N. Johnstone, A.S. Eggemen, D. Hunert, M.C. Hardy, C.M.F. Rae, and P.A. Midgley: Acta Mater., 2017, vol. 130 , pp. $271-80$.

7. A.S. Wilson: Mater. Sci. Technol., 2016, vol. 33, pp. 1108-18.

8. J.X. Yang, Q. Zheng, X.F. Sun, H.R. Guan, and Z.Q. Hu: Scr. Mater., 2006, vol. 55, pp. 331-34.

9. E04 Committee: Standard Practice for Electrolytic Extraction of Phases from Ni and Ni-Fe Base Superalloys Using a Hydrochloric-Methanol Electrolyte, ASTM International, 2010.

10. M.J. Donachie, O.H. Kriege: Phase Extraction and Analysis in Superalloys-Summary of Investigations by ASTM Committee E4 Task Group 1, 1972.
11. H.S. Lee, D.S. Kim, K.B. Yoo, and K.S. Song: Met. Mater. Int., 2012, vol. 18, pp. 287-93.

12. D.W. Hunt: Stability \& mechanical properties of a nickel-base turbine disc alloy, University of Cambridge, Cambridge, 2001.

13. S.E. Kim, M.P. Jackson, R.C. Reed, C. Small, A. James, and N.K. Park: Mater. Sci. Eng. A, 1998, vol. 245A, pp. 225-32.

14. R.J. Mitchell, C.M.F. Rae, and S. Tin: Mater. Sci. Technol., 2005, vol. 21, pp. 125-32.

15. R.A. Mitchell: Development of a New Powder Processed Ni-Base Superalloy for Rotor Disc Application, University of Cambridge, Cambridge, 2004.

16. A.J. Manning: Development of a Polycrystalline Ni Base Superalloy for Gas Turbine Disc Applications, University of Cambridge, Cambridge, 1999.

17. A.A. Coelho: TOPAS-Academic. Version 5. 2012, http://topas-ac ademic.net/.

18. J.O. Andersson, T. Helander, L. Hoglund, P.F. Shi, and B. Sundman: Calphad, 2002, vol. 26, pp. 273-312.

19. Thermo-Calc Software, Thermotech Ni-based Superalloys Database version 8.0.

20. N.S. Stoloff: Int. Met. Rev., 1989, vol. 34 (4), pp. 153-83.

21. D. Tytko, P. Choi, J. Klower, A. Kostka, G. Inden, and D. Raabe: Acta Mater., 2012, vol. 60, pp. 1731-40.

22. N.I. Medvedeva, D.C. Van Aken, and J.E. Medvedeva: Comput. Mater. Sci., 2015, vol. 96, pp. 159-64.

23. H.L. Yakel: Acta Crystallogr., 1983, vol. B39, pp. 20-28.

24. H.L. Yakel: Acta Crystallogr., 1983, vol. B39, pp. 28-33.

25. J.M. Joubert: Prog. Mater. Sci., 2008, vol. 53, pp. 528-83.

26. E04 Committee: Test Methods for Determining Average Grain Size, ASTM International, 2014.

Publisher's Note Springer Nature remains neutral with regard to jurisdictional claims in published maps and institutional affiliations. 